\title{
Rigorous bounds for seismic dispersion and attenuation due to wave-induced fluid flow in porous rocks
}

\author{
Boris Gurevich ${ }^{1}$ and Dina Makarynska ${ }^{2}$
}

\begin{abstract}
The Hashin-Shtrikman (HS) bounds define the range of bulk and shear moduli of an elastic composite, given the moduli of the constituents and their volume fractions. Recently, the HS bounds have been extended to the quasi-static moduli of composite viscoelastic media. Because viscoelastic moduli are complex, the viscoelastic bounds form a closed curve on the complex plane. We analyze these general viscoelastic bounds for a particular case of a porous solid saturated with a Newtonian fluid. In our analysis, for poroelastic media, the viscoelastic bounds for the bulk modulus are represented by a semicircle and a segment of the real axis, connecting formal HS bounds that are computed for an inviscid fluid. Importantly, viscoelastic bounds for poroelastic media turn out to be independent of frequency. However, because the bounds are quasi-static, the frequency must be much lower than Biot's characteristic frequency. Furthermore, we find that the bounds for the bulk modulus are attainable (realizable). We also find that these viscoelastic bounds account for viscous shear relaxation and squirt-flow dispersion, but do not account for Biot's global flow dispersion, because the latter strongly depends on inertial forces.
\end{abstract}

\section{INTRODUCTION}

It is generally believed that seismic attenuation and dispersion in many fluid-saturated porous rocks are controlled by the viscosity of the pore fluid. When an elastic wave propagates through a fluidsaturated medium, it creates local pressure gradients within the fluid phase, resulting in fluid flow and corresponding viscous dissipation until the pore pressure is equilibrated. This process can take different forms depending on the spatial scale of the pressure gradients and the geometry of the pore space. Commonly identified mechanisms of wave-induced fluid flow are global or macroscopic flow, local or squirt flow, and mesoscopic flow (Batzle et al., 2006; Müller et al., 2010). In the global flow, wave-induced pressure gradients are caused by the difference in solid and fluid densities (Biot, 1956a, 1956b; Pride et al., 1992). Squirt flow occurs because different pores have different compliances, and thus the same stress in the wave causes different deformation of different pores (or different parts of the same pore), which in turn creates fluid pressure gradients on the pore scale (Mavko and Nur, 1975, 1979; O'Connell and Budiansky, 1977; Jones, 1986; Dvorkin et al., 1995; Chapman et al., 2002; Pride et al., 2004; Gurevich et al., 2010). Mesocopic flow is similar in nature to squirt flow, but is caused by pressure gradients between areas of the rock which are much larger than the pore scale but much smaller than the wavelength (White, 1975; Dutta and Ode, 1979a, 1979b; Lopatnikov and Gurevich, 1988; Gurevich and Lopatnikov, 1995; Pride et al., 2003; Müller et al., 2010). These pressure gradients are caused by spatial variations of rock matrix compliance (White et al., 1975) or in pore fluid compressibility (White, 1975; Gist, 1994; Johnson, 2001; Toms et al., 2007).

Common to all three dissipation mechanisms described above is the fact that they are all related to fluid viscosity. Indeed, none of these mechanisms occurs in porous media saturated with an inviscid fluid. Despite this common feature, there is as yet no unified theoretical model of all these mechanisms. Therefore, it appears particularly useful to investigate rigorous bounds for the dispersion and attenuation that would be independent of the geometry. In the theory of elasticity, the Hashin-Shtrikman (HS) bounds define the range of bulk and shear moduli of an elastic composite, given the moduli of the constituents and their volume fractions. Recently, the HS bounds have been extended to the moduli of composite viscoelastic media (Gibiansky and Milton, 1993; Milton and Berryman, 1997). In this paper, we aim to explore these bounds for a particular case of a porous medium, that is, a mixture of an elastic solid and a linear Newtonian fluid.

\footnotetext{
Manuscript received by the Editor 2 February 2012; revised manuscript received 29 May 2012; published online 18 September 2012.

${ }^{1}$ Curtin University, Perth, Western Australia. E-mail: B.Gurevich@ curtin.edu.au.

${ }^{2}$ CSIRO Earth Science and Resource Engineering, Perth, Western Australia. E-mail: dgmakarynska@ovi.com. (C) 2012 Society of Exploration Geophysicists. All rights reserved.
} 


\section{RIGOROUS VISCOELASTIC BOUNDS}

The HS bounds define the range of bulk and shear moduli of an elastic composite, given the moduli of the constituents and their volume fractions (Hashin and Shtrikman, 1963; Christensen, 1979; Mavko et al., 1998). Gibiansky and Milton (1993) extended the HS bounds for bulk modulus to linear viscoelasticity using variational principles. They considered the response of statistically isotropic 2D and 3D composites with two viscoelastic isotropic phases mixed in fixed proportions in the quasi-static regime. Note that in the frequency domain (that is, for strains and stresses that are sinusoidal functions of time), a viscoelastic solid is described by the same equations as an elastic solid, but with moduli that are complex-valued, with the real part defining the wave velocity and the imaginary part corresponding to attenuation (Hashin, 1970; Christensen, 1971). Therefore, for a given frequency, the bounds should be represented by a closed curve in the complex plane that encircles the permissible region for the (complex) values of the moduli. This closed curve consists of arcs of circles containing several points related to the bulk and shear moduli of the constituents. The resulting bounds form a lens-shaped region obtained by taking the intersection of all such arcs. Similarly to the HS bounds, the viscoelastic bounds are independent of the microstructure of the rock. In the limiting case of an elastic medium, all the constituent moduli are real and the viscoelastic bounds reduce to the elastic HS bounds.

For a composite of two viscoelastic media with bulk and shear moduli $K_{1}, G_{1}, K_{2}$, and $G_{2}$, respectively, Gibiansky and Milton (1993) give their main result as follows. Let $\operatorname{Arc}\left(a_{1}, a_{2}, a_{3}\right)$ denote the arc of a circle in the complex plane joining the points $a_{1}$ and $a_{2}$ that when extended passes through $a_{3}$. For the threedimensional complex bulk modulus bounds, consider four arcs: $\operatorname{Arc}\left(K_{1^{*}}, K_{2^{*}}, K_{h}\right), \quad \operatorname{Arc}\left(K_{1^{*}}, K_{2^{*}}, K_{a}\right), \quad \operatorname{Arc}\left(K_{1^{*}}, K_{2^{*}}, K_{1}\right), \quad$ and $\operatorname{Arc}\left(K_{1^{*}}, K_{2^{*}}, K_{2}\right)$. Then, the outermost pair of these arcs will give us the bounds.

In the above expressions,

$$
\begin{gathered}
K_{1 *}=f_{1} K_{1}+f_{2} K_{2}-\frac{f_{1} f_{2}\left(K_{1}-K_{2}\right)^{2}}{f_{2} K_{1}+f_{1} K_{2}+G_{1}}, \\
K_{2 *}=f_{1} K_{1}+f_{2} K_{2}-\frac{f_{1} f_{2}\left(K_{1}-K_{2}\right)^{2}}{f_{2} K_{1}+f_{1} K_{2}+G_{2}}, \\
K_{a}=f_{1} K_{1}+f_{2} K_{2},
\end{gathered}
$$

and

$$
K_{h}=\left(f_{1} / K_{1}+f_{2} / K_{2}\right)^{-1},
$$

where $f_{1}$ and $f_{2}=1-f_{1}$ are volume fractions of the two viscoelastic constituents. Expressions 1 and 2 formally coincide with the HS

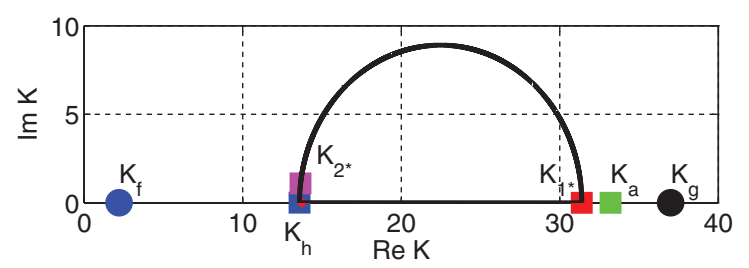

Figure 1. Bounds for the complex bulk modulus (solid lines). bulk modulus bounds, whereas equations 3 and 4 formally coincide with the upper and lower Voigt-Reuss-Hill bounds (Christensen, 1979; Mavko et al., 1998). However, for viscoelastic media, these expressions are not bounds. In viscoelastic media, the moduli also are frequency-dependent, and therefore the given bounding region may vary with the frequency.

\section{BOUNDS FOR A POROUS MEDIUM}

A fluid-saturated porous medium (also called a poroelastic medium) is a mixture of an elastic solid and a viscous fluid, and therefore represents a special case of a viscoelastic composite. We use the term "poroelastic" in a broad sense, without assuming anything about the connectivity of the solid or fluid phase. Consider that the elastic frame is composed of a single linearly elastic material with real moduli $K_{1}=K_{g}$ and $G_{1}=G_{g}$, and the fluid is characterized by a bulk modulus $K_{2}=K_{f}$ and a viscosity $\eta$, so that at frequency $\omega$ its complex shear modulus is $G_{2}=i \omega \eta$. We further assume that the imaginary shear modulus of the fluid $G_{2}=i \omega \eta$ is much smaller than the real part of the bulk modulus: $\omega \eta \ll \operatorname{Re} K_{2}$. This condition is valid for most fluids even at ultrasonic frequencies. For instance, for water at $1 \mathrm{MHz}, \omega \eta=6.3$. $10^{3} \mathrm{~Pa}$ whereas $\operatorname{Re} K_{2}=2.25 \cdot 10^{9} \mathrm{~Pa}$. Furthermore, if the bulk and shear viscosities of the fluid are of the same order of magnitude, the effect of the bulk viscosity on the fluid bulk modulus $K_{2}$ is negligible, and thus it can be assumed that $K_{2}$ is real. We further assume that the solid material is stiffer than the fluid, $K_{1}>K_{2}$. Indeed, the least compressible pore fluid (brine) has a bulk modulus of at most $3 \mathrm{GPa}$, whereas most minerals have a bulk modulus over $10 \mathrm{GPa}$ (Mavko et al., 1998). Solid and fluid displacements are assumed small, so that equations of linear viscoelasticity apply.

If we now examine expressions $1-4$, we will see that the moduli $K_{1^{*}}, K_{a}$, and $K_{h}$, as well as moduli $K_{1}$ and $K_{2}$ are all real, whereas the modulus $K_{2^{*}}$ is complex with a very small imaginary part. Expanding the expression for $K_{2^{*}}$ in the powers of a small (by absolute value) quantity $G_{2}=i \omega \eta$, we can rewrite equation 2 in the form

$$
K_{2 *}=f_{1} K_{1}+f_{2} K_{2}-\frac{\left(K_{1}-K_{2}\right)^{2}}{\frac{K_{1}}{f_{1}}+\frac{K_{2}}{f_{2}}}\left(1-\frac{G_{2}}{\frac{K_{1}}{f_{1}}+\frac{K_{2}}{f_{2}}}\right),
$$

or

$$
K_{2 *}=K_{h}+\left(\frac{K_{1}-K_{2}}{K_{1} / f_{1}+K_{2} / f_{2}}\right)^{2} G_{2} .
$$

Thus, $\operatorname{Re} K_{2^{*}}=K_{h}$ and on the complex plane $K_{2^{*}}$ lies very close to $K_{h}$ but slightly off the real axis. The construction of the bounds is illustrated in Figure 1 for a mixture of quartz $\left(K_{1}=37 \mathrm{GPa}\right.$ and $\left.G_{1}=44 \mathrm{GPa}\right)$ and water $\left(K_{2}=2.2 \mathrm{GPa}\right.$ and $\left.\eta=0.001 \mathrm{~Pa} \cdot \mathrm{s}\right)$, and for porosity $\phi=f_{2}=0.109$ and frequency $f=4 \cdot 10^{10} \mathrm{~Hz}$. Such an extremely high frequency was chosen to see the deviation of the modulus $K_{2^{*}}$ from the real axis. For frequencies below $1 \mathrm{MHz}, K_{2^{*}}$ would always be directly above $K_{h}$, but would be visually indistinguishable from it. We also note that the moduli $K_{a}$, $K_{h}$, and $K_{1^{*}}$ represent the true Voigt, Reuss, and upper HS bounds for the (real) bulk modulus of a mixture of a solid with the bulk and shear moduli $K_{1}$ and $G_{1}$, and an inviscid fluid with the bulk modulus $K_{2}<K_{1}$. Thus, $K_{2}<K_{h}<K_{1^{*}}<K_{a}<K_{1}$. It follows that the arcs $\operatorname{Arc}\left(K_{1^{*}}, K_{2^{*}}, K_{a}\right), \operatorname{Arc}\left(K_{1^{*}}, K_{2^{*}}, K_{1}\right)$, and $\operatorname{Arc}\left(K_{1^{*}}, K_{2^{*}}, K_{2}\right)$ are almost straight line segments coinciding with the real axis, whereas the $\operatorname{arc} \operatorname{Arc}\left(K_{1^{*}}, K_{2^{*}}, K_{h}\right)$ is a semicircle connecting $K_{h}$ and $K_{1^{*}}$. 
The bounding region is a half-disk between this semicircle and the real axis.

We also note that the real moduli $K_{1}, K_{2}, K_{h}, K_{1^{*}}$, and $K_{a}$ are independent of frequency, whereas $K_{2^{*}}$ has a constant real part and a frequency dependent imaginary part. However, as long as this imaginary part remains small, the circular $\operatorname{arc} \operatorname{Arc}\left(K_{1^{*}}, K_{2^{*}}, K_{h}\right)$ remains the same. Thus, we can conclude that the bulk modulus bounds for a poroelastic medium are the same for all frequencies.

A similar (but somewhat more involved) derivation shows that the shear modulus bounds for a poroelastic medium also define a half-disk in a complex plane. This derivation is based on the expressions for shear modulus bounds for a general viscoelastic composite obtained for isotropic 3D composites by Milton and Berryman (1997).

\section{PROPERTIES OF THE BOUNDS}

\section{Quasi-static nature of the bounds}

At this point, we recall that all the bounds discussed in this paper are quasi-static. Indeed, the HS bounds for elastic media are derived from equations of static elasticity and represent the static composite moduli, that is, moduli in the limit of zero frequency. Dynamic effects, such as scattering attenuation and dispersion caused by the composite's microstructure, are not accounted for by these bounds. However, the concept of the static limit is not useful for viscoelastic media because the constituent moduli themselves are frequency dependent. Therefore, in viscoelasticity, moduli are called quasi-static.

For a homogeneous viscoelastic solid, the quasi-static state is the state that is adequately described by equations of quasi-static viscoelasticity, that is, viscoelasticity equations without the inertial terms (Christensen, 1971). For composite viscoelastic media, an additional constraint is that strains and stresses are locally statistically homogeneous (Hashin, 1970). This condition is satisfied when the wavelength (of all wave modes that may propagate in such a medium) is much larger than the typical size of heterogeneities.

Viscoelastic materials used in engineering mechanics and construction engineering are typically nearly elastic solids, that is, solids whose bulk and shear moduli are complex but with an imaginary part much smaller than the real part. In a poroelastic medium, one of the constituents is a fluid, often a low-viscosity fluid with a very small complex shear wave (viscous wave) velocity, and hence has a very small wavelength. Application of the viscoelastic bounds to such a medium requires that the wavelength of the viscous wave in the pore fluid, also known as the viscous skin depth (Biot, 1956b; Johnson et al., 1987; Gurevich, 2002), be large compared to the heterogeneity size. Therefore, the range of frequencies in which the viscoelastic bounds are valid is much more restrictive than for near-elastic composites and requires careful analysis.

To understand the range of validity of the bounds, let us examine the processes that cause wave attenuation and dispersion in poroelastic media. Of the known dissipation mechanisms, it is obvious that standard viscoelastic relaxation (Christensen, 1971; Gurevich, 2002) must obey the bounds because it can be modeled by applying the effective medium theory for elastic media to complex viscoelastic moduli (Hashin, 1970). At the same time, the bounds would not be valid for the scattering attenuation because in the elastic limit these bounds reduce to HS bounds, which are static and do not account for scattering.
What about the wave-induced flow? Below, we contend that attenuation and dispersion caused by the squirt flow must obey the bounds, whereas the global flow should not.

\section{Squirt flow}

Squirt flow is the flow of the pore fluid from more compliant voids (such as cracks or compliant grain contacts) to stiffer pores and vice versa. To illustrate this process, we propose the following artificial porous medium. Assume that the matrix of this porous medium at a given porosity is a Hashin's coated spheres assemblage (Hashin, 1962; Christensen, 1971). This structure is known to be the stiffest possible structure of a porous medium with a given porosity, and its bulk modulus is given by the upper HS bound. In the dry state, we denote this modulus $K_{\mathrm{HS}}$. Let this structure be permeated by a set of thin cracks such that their overall volume is negligible, but the aspect ratio is very small and compliance large. The cracks are thin but large in diameter and thus intersect many pores. Because their total volume is negligible, the cracks have no influence on the upper HS bound, which is still equal to $K_{\mathrm{HS}}$. However, by making their aspect ratio as small as possible, we can make the lower HS bound as small as we like (because the dry porous medium is a mixture of a solid and a vacuum, its lower HS bound is zero).

Now consider this matrix saturated with a Newtonian fluid. At low frequencies, its bulk modulus $K_{\text {low }}$ is given by Gassmann's (1951) equation

$$
\frac{1}{K_{\text {low }}}=\frac{1}{K_{1}}+\frac{f_{2}\left(\frac{1}{K_{2}}-\frac{1}{K_{1}}\right)}{1+f_{2}\left(\frac{1}{K_{2}}-\frac{1}{K_{1}}\right) /\left(\frac{1}{K_{\text {dry }}}-\frac{1}{K_{1}}\right)}
$$

where $K_{\text {dry }}$ is the dry bulk modulus. Because this dry modulus is close to zero, Gassmann's equation reduces to Wood's equation,

$$
K_{\text {low }} \approx\left(f_{1} / K_{1}+f_{2} / K_{2}\right)^{-1},
$$

and thus the saturated modulus is close to $K_{\text {low }} \approx K_{h}$. However, at high frequencies, the fluid has no time to move between pores and cracks, and thus the bulk modulus is close to the upper HS bound, $K_{\text {high }} \approx K_{1^{*}}$ (Mavko and Jizba, 1991; Gurevich et al., 2009). At intermediate frequencies, the modulus will correspond to a point on a continuous curve connecting the points $K_{\text {low }}=K_{h}$ and $K_{\text {high }}=K_{1^{*}}$.

As mentioned earlier, there is no universally accepted model of squirt flow. To illustrate the effect of squirt, we use the model recently proposed by Gurevich et al. (2010). To model the frequency dependency of the moduli, Gurevich et al. (2010) assume the geometrical configuration proposed by Murphy et al. (1986): a compliant pore forms a disk-shaped gap between two grains, and its edge opens into a toroidal stiff pore. Figure 2 shows the prediction of this model for the bulk and shear moduli of hypothetical rock described above, with crack (intergranular gap) aspect ratio of $10^{-3}$, along with the corresponding viscoelastic bounds. Different red curves correspond to different crack porosities ranging from $10^{-6}$ to $10^{-2}$. Different curves may be thought of as corresponding to different effective pressures (Shapiro, 2003; Gurevich et al., 2010; Pervukhina et al., 2010). Different points on each curve correspond to different frequencies; frequency increases as the point moves along the curve clockwise. The corresponding frequency dependencies of the real part of the moduli 
and dimensionless attenuation factors $1 / Q$ for the bulk and shear modulus are shown in Figures 3 and 4, respectively. On the complex planes $\operatorname{Re} K, \operatorname{Im} K$ and $\operatorname{Re} G, \operatorname{Im} G$ in Figure $2 \mathrm{a}$ and $2 \mathrm{~b}$, the curves look like semicircles and indeed they are. Carcione and Gurevich (2011) have shown that the bulk modulus given by the squirt model of Gurevich et al. (2010) can be written in the form of a Zener element

$$
K(\omega)=K_{G}\left(\frac{1+i \omega \tau_{a}}{1+i \omega \tau_{b}}\right)
$$

where $K_{G}$ is the zero-frequency (Gassmann) modulus, $\tau_{a}$ and $\tau_{b}$ are real-valued constants that can be calculated from the properties of the medium, and $\tau_{a} / \tau_{b}=K_{1 *} / K_{G}$. On the plane $\operatorname{Re} K, \operatorname{Im} K$, the curve given by equation 9 is a semicircle with a center at a midpoint $K_{c}$ between $K_{G}$ and $K_{1^{*}}: K_{c}=\left(K_{G}+K_{1 *}\right) / 2$. Indeed, the squared distance between $K(\omega)$ given by equation 9 and $K_{c}$ is $\left|K(\omega)-K_{\mathrm{c}}\right|^{2}=K_{G}^{2}\left(\tau_{a}-\tau_{b}\right)^{2} / 4 \tau_{b}^{2}$, and is independent of frequency. Thus, equation 9 describes a semicircle with the center at $K_{c}=\left(K_{G}+K_{1 *}\right) / 2$ and a radius $R=K_{G}\left(\tau_{a}-\tau_{b}\right)^{2}\left(2 \tau_{b}\right)^{-1}=$ $\left(K_{1 *}-K_{G}\right) / 2$. For cracks with a very small aspect ratio, $K_{G} \approx K_{h}$, and thus the semicircle given by equation 9 and corresponding to the squirt-flow model of Gurevich et al. (2010) coincides with the semicircle forming one of the arcs of the viscoelastic bound.

The above analysis yields two important conclusions. First, the squirt flow attenuation and dispersion obey the viscoelastic bounds. Second, viscoelastic bounds for the bulk modulus of a poroelastic media are attainable (realizable), that is, for each point within the bounds, there exists a geometrical configuration for which this point corresponds to the exact value of the bulk modulus. No such conclusion could be drawn for the shear modulus.

a)

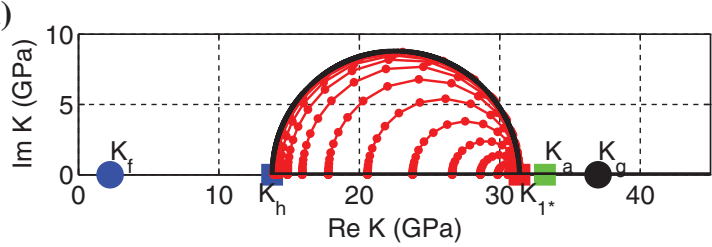

b)

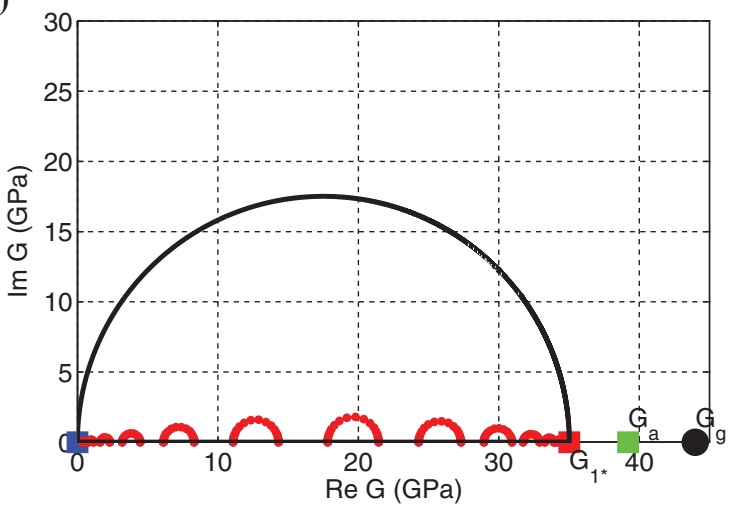

Figure 2. Bounds for the complex bulk (a) and shear (b) modulus (solid black lines), and predictions based on the squirt model (red lines); larger semicircles correspond to larger compliant porosity. Different points on the same red semicircle correspond to different frequencies. Symbols represent different moduli described in the text.
Note that in the calculation of the squirt-flow dispersion we have taken the bulk and shear moduli of the nonfractured dry medium to be equal to the upper HS bounds for the respective moduli. It is a classical result that Hashin's coated spheres assemblage attains the upper HS bounds for the bulk modulus; however, it is not known whether this also is true for the shear modulus. Thus, the shear modulus of the Hashin assemblage (Figure 2b) could be slightly lower than the upper HS shear modulus bound $G_{1^{*}}$, which is given by

$$
G_{1 *}=G_{1}+\frac{f_{2}}{-G_{1}^{-1}+2 f_{1}\left(K_{1}+2 G_{1}\right) /\left[5 G_{1}\left(K_{1}+4 G_{1} / 3\right)\right]} .
$$

This would not affect the conclusions of this section. Alternatively, we could have instead used a high-rank laminate structure, which does attain the HS bounds for bulk and shear moduli (Francfort and Murat, 1986; Milton, 2002; Torquato, 2002).

\section{Global (Biot) flow}

Global (or macroscopic) flow is the pore fluid flow (relative to the solid) caused by pressure gradients between peaks and troughs of the wave. This flow is called global because it occurs on the scale of

a)

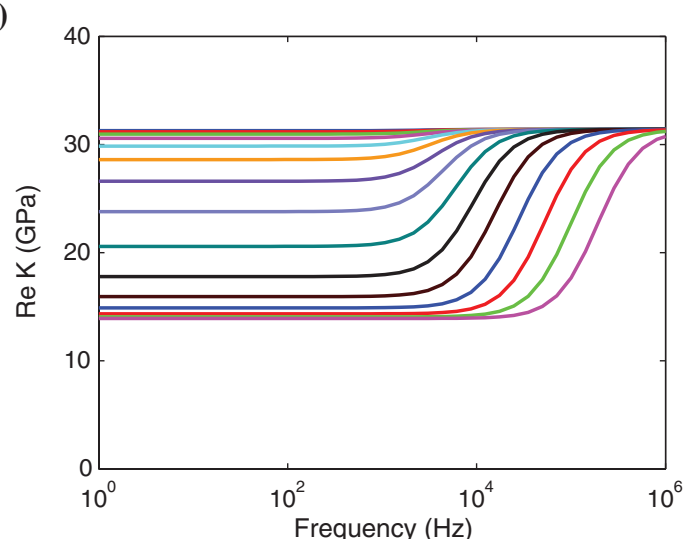

b)

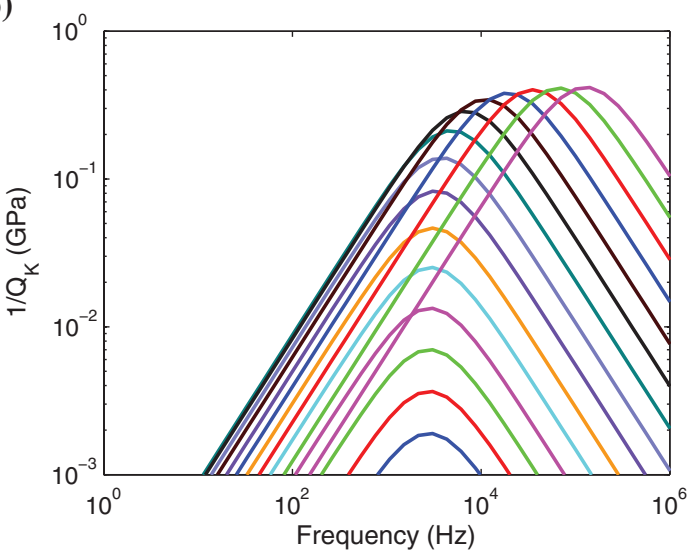

Figure 3. Frequency dependency of the real part (a) and inverse quality factor (b) of the bulk modulus of the hypothetical rock described in the text. Different lines correspond to different levels of compliant porosity. 
wavelength, and in rocks, the wavelength of a seismic wave is usually very large compared to the size of individual pores.

Attenuation and dispersion of elastic waves due to the global flow is described by Biot's theory of poroelasticity (Biot, 1956a, 1956b; Pride et al., 1992; Müller et al., 2010). In Biot's theory, low- and high-frequency limits of the moduli are real. Therefore, as with squirt, dispersion on the complex plane is represented by a continuous curve connecting two points located on the real axis. To examine whether the global flow attenuation obeys the bounds, we first explore these low- and high-frequency limits. We do this for the shear modulus (not the bulk modulus) because it is given by simpler expressions.

Let the rock matrix be an elastic solid permeated by a random bundle of identical cylindrical tubes with circular cross-section and random distribution of orientations (see, e.g., Johnson et. al., 1987). We further assume that the overall porosity of this system of tubes $\phi=f_{2}$ is small $(\phi \ll 1)$. Then, the dry modulus can be computed with a low-concentration effective medium approximation for tubes or needles (Küster and Töksöz, 1974; Berryman, 1980). In accordance with Gassmann's and Biot's theory, the low-frequency saturated modulus is equal to the dry modulus. The result has the form

$$
G_{\text {low }}=G_{\text {dry }}=G_{1}(1-B \phi),
$$

where the coefficient $B$ depends on the Poisson's ratio of the solid. The modulus given by equation 11 is close to, but lower than, the upper HS bound $G_{1^{*}}$ as given by equation 10 because the tubular structure is less stiff than the structure with the same volume concentration of spheres (Christensen, 1979). If we write $G_{1^{*}}$ in a similar form $G_{1 *}=G_{1}(1-C \phi)$, then it follows that $C<B$.

The high-frequency shear modulus can be computed from the solution of Biot's dispersion equation for the shear wave velocity (Johnson and Plona, 1982; Mavko et al., 1998)

$$
V_{\mathrm{S}, \text { high }}^{2}=\frac{G_{\mathrm{dry}}}{\left(\rho-\phi \rho_{2} \alpha^{-1}\right)},
$$

where $\rho=(1-\phi) \rho_{1}+\phi \rho_{2}$ is the overall density of the saturated medium, $\rho_{1}$ and $\rho_{2}$ are densities of the solid and fluid respectively, and $\alpha$ is the tortuosity, which is equal to three for a system of randomly oriented circular tubes (Torquato, 2002). Thus, the highfrequency shear modulus is

$$
G_{\mathrm{high}}=\frac{G_{\mathrm{dry}} \rho}{\rho-\phi \rho_{2} \alpha^{-1}} \approx \frac{G_{1}(1-B \phi)}{1-\frac{\phi r}{\alpha(1-\phi(1-r))}},
$$

where $r=\rho_{2} / \rho_{1}$. Equation 13 shows that the high-frequency limit of the shear modulus is controlled by the density ratio $r$, and can be larger than the upper HS bound if $r$ is sufficiently large. The largest value of $G_{\text {high }}$ is attained for large $r$ and is $G_{\text {high }}^{\max }=(3 / 2) G_{1}$ $(1-B \phi)$. Very large $r$ means fluid density is much higher than solid density. This is an unusual, but not impossible, situation; it can occur e.g., when the solid itself contains isolated and empty pores. For small porosity, modulus $G_{\text {high }}^{\max }=(3 / 2) G_{1}(1-B \phi)$ is higher than the solid modulus $G_{1}$, let alone the upper HS bound $G_{1^{*}}$. This result may sound counterintuitive. The physical explanation is that at high frequencies, the fluid and solid movements are no longer coupled by fluid viscosity, and thus the shear (and compressional) wave only has to "move" the mass of the frame plus the added fluid mass trapped by the tortuosity of the pores. This results in a larger modulus than at low frequencies, in which the solid and fluid are "glued" to each other by viscous forces and the wave has to move the overall mass of the solid and fluid. In fact, the presence of densities in equations 12 and 13 indicates that Biot's dispersion is controlled by inertial forces, which are not accounted for in the equations of quasi-static viscoelasticity, and thus do not have to obey the quasi-static viscoelastic bounds. Thus, we can conclude that the viscoelastic bounds can only be used for frequencies much smaller than Biot's characteristic frequency.

It also is clear that the bounds should be valid for dispersion and attenuation due to mesoscopic flow, because it has the same physical nature as squirt flow (but on different spatial scales), and because it can be described by the low-frequency version of Biot's theory of poroelasticity. We should note however that global flow and mesoscopic flow require solid and fluid to be interconnected so as to form an interpenetrating continua. If the pores are isolated, the low-frequency restriction would not apply (note that in this case the permeability of the medium is zero and thus Biot's characteristic frequency is infinity).

a)

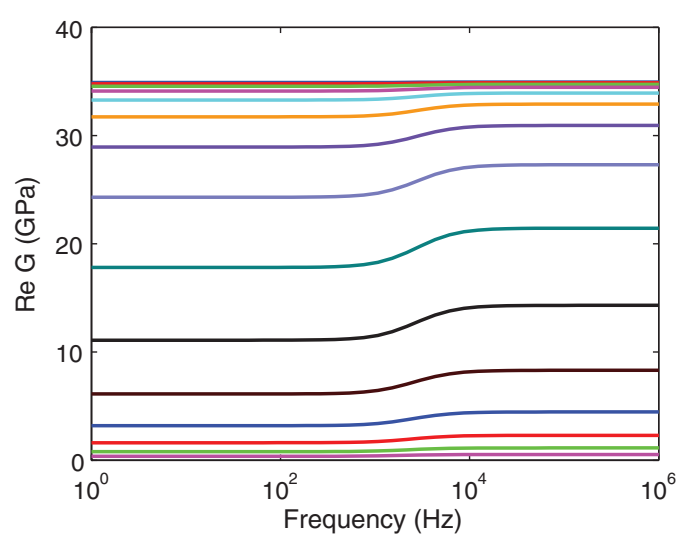

b)

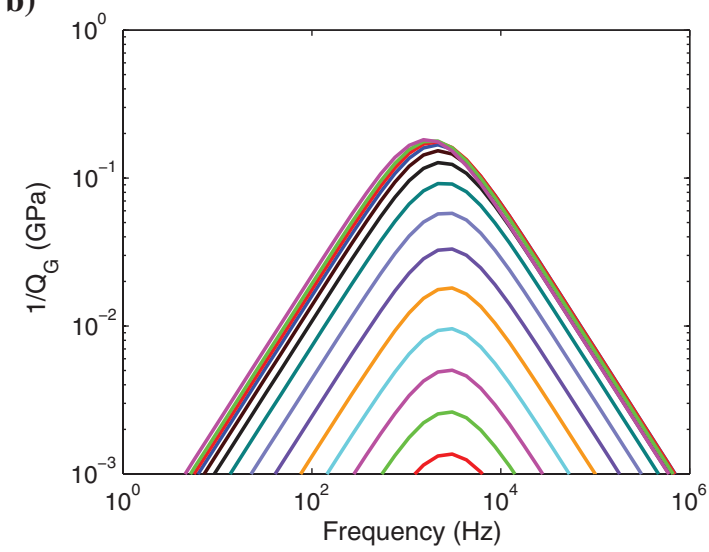

Figure 4. Frequency dependency of the real part (a) and inverse quality factor (b) of the shear modulus of the hypothetical rock described in the text. Different lines correspond to different levels of compliant porosity. 


\section{DISCUSSION}

The bounds described in this paper correspond to complex bulk and shear moduli in the frequency domain. Thus, they most directly apply to moduli obtained in stress-strain measurements on rock samples in which the driving stress oscillations are sinusoidal in time (Spencer, 1981; Paffenholz and Burkhardt, 1989; Batzle et al; 2006; Mikhaltsevitch et al., 2011; Takei et al., 2011; Boitnott et al., 2011). In such experiments, the ratio of stress to strain amplitude defines the absolute value of the complex elastic modulus, whereas the phase shift defines its argument. Because the viscoelastic bounds are an extension of the static elastic bounds to finite frequencies (and revert to elastic HS bounds in the low-frequency and low-viscosity limits), the corresponding experiment should be similar to that on elastic samples. This means that our bounds apply to the moduli measured with no-flow boundary conditions (as if the sample of the porous medium were enclosed in an elastic medium), that is, to the so-called undrained moduli (Biot and Willis, 1957). We also note that the undrained moduli control the velocity of the fast compressional wave and the shear wave. Thus, the moduli corresponding to the fast compressional and shear waves should obey the bounds. In the geophysical context, the bounds should apply to seismic and sonic frequencies but not to ultrasonic sub-MHz frequencies employed in laboratory experiments because the wave frequency must be much lower than Biot's characteristic frequency, which is typically between $0.1-1 \mathrm{MHz}$.

One interesting result of this study is that for a porous fractured medium with a particular geometry (Hashin's composite sphere geometry permeated by a set of fractures of infinitesimal crack density and infinitesimal aspect ratio), the complex bulk modulus given by the squirt flow model coincides with the rigorous bound obtained from very general mixture principles. We find this rather remarkable, given that the squirt flow model considers the fluid flow explicitly in a particular geometry, whereas the bound is based on the representation of the pore fluid as a viscoelastic solid with an imaginary shear modulus $G_{2}=i \omega \eta$. We interpret this as a consequence of the fact that the frequency domain representation $G_{2}=i \omega \eta$ gives an adequate description of fluid flow (in the limit of small displacements). We also conclude that the squirt flow model based on deformation-induced flow between a flat intergranular gap (or crack) and a stiff pore (Murphy et al., 1986; Gurevich et al., 2010; Carcione and Gurevich, 2011) gives the largest possible dissipation of all possible geometrical configurations (for bulk deformations). It would be interesting to compare the bounds to other theoretical models of squirt flow. Such analysis is beyond the scope of this paper.

Although we illustrated the performance of the bounds mainly with one squirt-flow model (Gurevich et al., 2010), the bounds also should apply to any model of dispersion/attenuation due to squirt or mesoscopic flow and, in particular, to the models mentioned in the introduction. The bounds also should apply to moduli computed numerically, either by solving equations of poroelasticity (see, e.g., Masson and Pride, 2007; Wenzlau and Müller, 2009; Carcione et al., 2010) or by direct pore-scale simulations (Saenger et al., 2011). As mentioned earlier, the bounds are restricted to isotropic porous media composed of one isotropic linearly elastic solid and one Newtonian fluid, and to frequencies well below Biot's characteristic frequency.

\section{CONCLUSIONS}

The HS bounds define the range of bulk and shear moduli of an elastic composite, given the moduli of the constituents and their volume fractions. Recently, the HS bounds have been extended to the quasi-static moduli of composite viscoelastic media. Because viscoelastic moduli are complex, the viscoelastic bounds form a closed curve on the complex plane. In this paper, we have explored the behavior of these general viscoelastic bounds for a particular case of a poroelastic medium. More specifically, we have examined the bounds for quasi-static viscoelastic moduli of a mixture of an elastic solid (represented by real bulk and shear moduli) and a Newtonian fluid (with a real bulk modulus and imaginary shear modulus) without making any assumptions about the geometry of such a mixture, or connectivity of the solid and fluid phases. Our analysis shows that for poroelastic media, the viscoelastic bounds for a bulk modulus are represented in the complex plane by a semicircle and a segment of the real axis, connecting the formal HS bounds (computed for an inviscid fluid). Furthermore, we have shown that these bounds are independent of frequency. That is, the entire dispersion curve, corresponding to the variation of the complex bulk modulus with frequency, stays within this bounding semicircular region.

We also have shown that the complex bulk modulus describing attenuation and dispersion due to squirt flow in a porous medium of a particular geometry spans the entire bounding region. This means that the bounds for the bulk modulus are attainable (realizable). That is, for any complex value $K$ within the bounding region on the complex plane, there exists a geometrical configuration of the porous medium such that at a certain frequency the complex bulk modulus of the fluid-saturated medium with such a microstructure equals $K$. No such conclusion has been obtained for the shear modulus. More research is required to determine whether the complex bounds for the shear modulus are attainable by any geometrical configurations.

We also have constructed another artificial example of a porous medium, for which it is easy to compute the magnitude of the shear modulus dispersion due to global flow (using Biot's theory). This calculation shows that for some combinations of rock and fluid properties, the high-frequency limit of the shear modulus lies outside the bounds. Therefore, the viscoelastic bounds account for the viscous shear relaxation and squirt-flow dispersion, but not for Biot's global flow dispersion. This is to be expected, because the bounds are quasi-static whereas the global flow dispersion is largely controlled by inertial forces.

\section{ACKNOWLEDGMENTS}

The authors thank Tobias M. Müller, Marina Pervukhina, and José Carcione for inspiring discussions, Robert J. Galvin for editing the manuscript, and the sponsors of the Curtin Reservoir Geophysics Consortium for financial support. Very special thanks go to Mark Chapman (University of Edinburgh) for presenting this work at the 2011 SEG Annual Meeting.

\section{REFERENCES}

Batzle, M. L., D.-H Han, and R. Hofmann, 2006, Fluid mobility and frequency-dependent seismic velocity - Direct measurements: Geophysics, 71, no. 1, N1-N9, doi: 10.1190/1.2159053.

Berryman, J. G., 1980, Long-wavelength propagation in composite elastic media II. Ellipsoidal inclusions: Journal of the Acoustical Society of America, 68, 1820-1831, doi: 10.1121/1.385172. 
Biot, M. A., 1956a, Theory of propagation of elastic waves in a fluid saturated porous solid. I. Lower frequency range: Journal of the Acoustical Society of America, 28, 168-178.

Biot, M. A., 1956b, Theory of propagation of elastic waves in a fluid saturated porous solid. II. Higher frequency range: Journal of the Acoustical Society of America, 28, 179-191.

Biot, M. A., and D. G. Willis, 1957, The elastic coefficients of the theory of consolidation: Journal of Applied Mechanics and Technical Physics, 24, 594-601.

Boitnott, G. N., M. K. Broadhead, and T. H. Keho, 2011, Laboratory measurements of modulus dispersion in sandstone at seismic frequencies: 81st Annual International Meeting, SEG, Expanded Abstracts, 2236-2240, doi: $10.1190 / 1.3627653$

Carcione, J. M., and B. Gurevich, 2011, Differential form and numerical implementation of Biot's poroelasticity equations with squirt dissipation: Geophysics, 76, no. 6, N55-N64, doi: 10.1190/geo2010-0169.1.

Carcione, J. M., C. Morency, and J. E. Santos, 2010, Computational poroelasticity - A review: Geophysics, 75, no. 5, 75A229-75A243, doi: 10 $.1190 / 1.3474602$.

Chapman, M., S. V. Zatsepin, and S. Crampin, 2002, Derivation of a microstructural poroelastic model: Geophysical Journal International, 151, 427-451, doi: 10.1046/j.1365-246X.2002.01769.x.

Christensen, R. M., 1971, Theory of viscoelasticity: Academic Press.

Christensen, R. M., 1979, Mechanics of composite materials: WileyInterscience.

Dutta, N. C., and H. Odé, 1979a, Attenuation and dispersion of compressional waves in fluid-filled porous rocks with partial gas saturation (White model) - Part I: Biot theory: Geophysics, 44, 1777-1788, doi: 10.1190/1 .1440938 .

Dutta, N. C., and H. Odé, 1979b, Attenuation and dispersion of compressional waves in fluid-filled porous rocks with partial gas saturation (White model) — Part II: Results: Geophysics, 44, 1789-1805, doi: 10.1190/1 1440939.

Dvorkin, J., G. Mavko, and A. Nur, 1995, Squirt flow in fully saturated rocks: Geophysics, 60, 97-107, doi: 10.1190/1.1443767.

Francfort, G., and F. Murat, 1986, Homogenization and optimal bounds in linear elasticity: Archive for Rational Mechanics and Analysis, 94, 307334, doi: 10.1007/BF00280908.

Gassmann, F., 1951, Über die Elastizität Poröser Medien: Vierteljahresschrift der Naturforschenden Gesellschaft, 96, 1-23.

Gibiansky, L. V., and G. W. Milton, 1993, On the effective viscoelastic moduli of two-phase media. I. Rigorous bounds on the complex bulk modulus: Proceedings of the Royal Society of London A, 440, 163-188.

Gist, G. A., 1994, Interpreting laboratory velocity measurements in partially gas-saturated rocks: Geophysics, 59, 1100-1109, doi: 10.1190/1.1443666.

Gurevich, B., 2002, Effect of fluid viscosity on elastic wave attenuation in porous rocks: Geophysics, 67, 264-270, doi: 10.1190/1.1451798.

Gurevich, B., and S. L. Lopatnikov, 1995, Velocity and attenuation of elastic waves in finely layered porous rocks: Geophysical Journal International, 121, 933-947, doi: 10.1111/j.1365-246X.1995.tb06449.x.

Gurevich, B., D. Makarynska, O. De Paula, and M. Pervukhina, 2010, A simple model for squirt-flow dispersion and attenuation in fluid-saturated granular rocks: Geophysics, 75, no. 6, N109-N120, doi: 10.1190/1.3509782.

Gurevich, B., D. Makarynska, and M. Pervukhina, 2009, Ultrasonic moduli for fluid-saturated rocks: Mavko-Jizba relations rederived and generalized: Geophysics, 74, no. 4, N25-N30, doi: 10.1190/1.3123802.

Hashin, Z., 1962, The elastic moduli of heterogeneous materials: ASME Journal of Applied Mechanics, 29, 143-150.

Hashin, Z., 1970, Complex moduli of viscoelastic composites - I. General theory and application to particulate composites: International Journal of Solids and Structures, 6, 539-552.

Hashin, Z., and S. Shtrikman, 1963, A variational approach to the theory of the elastic behaviour of multiphase materials: Journal of Mechanics and Physics of Solids, 11, 127-140.

Johnson, D. L., 2001, Theory of frequency dependent acoustics in patchysaturated porous media: Journal of the Acoustical Society of America, 110, 682-694.

Johnson, D. L., J. Koplik, and R. Dashen, 1987, Theory of dynamic permeability and tortuosity in fluid-saturated porous media: Journal of Fluid Mechanics, 176, 379-402, doi: 10.1017/S0022112087000727.

Johnson, D. L., and T. J. Plona, 1982, Acoustic slow waves and the consolidation transition: Journal of the Acoustical Society of America, $\mathbf{7 2}$ $556-565$

Jones, T., 1986, Pore fluids and frequency-dependent wave propagation in rocks: Geophysics, 51, 1939-1953, doi: 10.1190/1.1442050.

Küster, G. T., and M. N. Toksöz, 1974, Velocity and attenuation of seismic waves in two-phase media: Part I: Theoretical formulations: Geophysics, 39, 587-606, doi: 10.1190/1.1440450.
Lopatnikov, S. L., and B. Gurevich, 1988, Transformational mechanism of elastic wave attenuation in saturated porous media: Izvestija Academy of Sciences USSR, Physics of the Solid Earth, 24, 151-154.

Masson, Y. J., and S. R. Pride, 2007, Poroelastic finite difference modeling of seismic attenuation and dispersion due to mesoscopic-scale heterogeneity: Journal of Geophysical Research, 112, B03204, doi: 10.1029/ 2006JB004592.

Mavko, G., and D. Jizba, 1991, Estimating grain-scale fluid effects on velocity dispersion in rocks: Geophysics, 56, 1940-1949, doi: 10.1190/1 .1443005 .

Mavko, G., T. Mukerji, and J. Dvorkin, 1998, The rock physics handbook: Tools for seismic analysis in porous media: Cambridge University Press.

Mavko, G., and A. Nur, 1975, Melt squirt in the aesthenosphere: Journal of Geophysical Research, 80, 1444-1448, doi: 10.1029/JB080i011p01444.

Mavko, G., and A. Nur, 1979, Wave attenuation in partially saturated rocks: Geophysics, 44, 161-178, doi: 10.1190/1.1440958.

Mikhaltsevitch, V., M. Lebedev, and B. Gurevich, 2011, A low-frequency laboratory apparatus for measuring elastic and anelastic properties of rocks: 81 st Annual International Meeting, SEG, Expanded Abstracts, 2256-2260, doi: 10.1190/1.3627657.

Milton, G. W., 2002, Theory of composites: Cambridge University Press. Milton, G. W., and J. G. Berryman, 1997, On the effective viscoelastic moduli of two-phase media. II. Rigorous bounds on the complex shear modulus in three dimensions: Proceedings of the Royal Society of London A, 453, $1849-1880$

Müller, T. M., B. Gurevich, and M. Lebedev, 2010, Seismic wave attenuation and dispersion resulting from wave-induced flow in porous rocks A review: Geophysics 75, no. 5, A147-A164, doi: 10.1190/1.3463417.

Murphy, W. F. I., K. W. Winkler, and R. L. Kleinberg, 1986, Acoustic relaxation in sedimentary rocks: Dependence on grain contacts and fluid saturation: Geophysics, 51, 757-766, doi: 10.1190/1.1442128.

O'Connell, R., and B. Budiansky, 1977, Viscoelastic properties of fluidsaturated cracked solids: Journal of Geophysical Research, 82, 57195735, doi: 10.1029/JB082i036p05719.

Paffenholz, J., and H. Burkhardt, 1989, Absorption and modulus measurements in the seismic frequency and strain range on partially saturated sedimentary rocks: Journal of Geophysical Research, 94, 9493-9507, doi: 10.1029/JB094iB07p09493.

Pervukhina, M., B. Gurevich, D. N. Dewhurst, and A. F. Siggins, 2010, Applicability of velocity-stress relationship based on the dual porosity concept to isotropic porous rocks: Geophysical Journal International, 181, $1473-1479$.

Pride, S. R., J. G. Berryman, and J. M. Harris, 2004, Seismic attenuation due to wave-induced flow: Journal of Geophysical Research, 109, B01201, doi: $10.1029 / 2003$ JB002639.

Pride, S. R., A. F. Gangi, and F. D. Morgan, 1992, Deriving the equations of motion for porous isotropic media: Journal of the Acoustical Society of America, 92, 3278-3290, doi: 10.1121/1.404178.

Pride, S. R., J. M. Harris, D. L. Johnson, A. Mateeva, K. T. Nihel, R. L. Nowack, J. W. Rector, H. Spetzler, R. Wu, T. Yamomoto, J. G. Berryman, and M. Fehler, 2003, Permeability dependence of seismic amplitudes: The Leading Edge, 22, 518-525, doi: 10.1190/1.1587671.

Saenger, E. H., F. Enzmann, Y. Keehm, and H. Steeb, 2011, Digital rock physics: Effect of fluid viscosity on effective elastic properties: Journal of Applied Geophysics, 74, 236-241.

Shapiro, S. A., 2003, Elastic piezosensitivity of porous and fractured rocks: Geophysics, 68, 482-486, doi: 10.1190/1.1567215.

Spencer, J. W., 1981, Stress relaxations at low frequencies in fluid-saturated rocks - Attenuation and modulus dispersion: Journal of Geophysical Research, 86, 1803-1812, doi: 10.1029/JB086iB03p01803.

Takei, Y., K. Fujisawa, and C. McCarthy, 2011, Experimental study of attenuation and dispersion over a broad frequency range 1 . The apparatus: Journal of Geophysical Research, 116, B09204.

Toms, J., T. M. Müller, and B. Gurevich, 2007, Seismic attenuation in porous rocks with random patchy saturation: Geophysical Prospecting, 55, 671678, doi: 10.1111/j.1365-2478.2007.00644.x.

Torquato, S., 2002, Random heterogeneous materials: Microstructure and macroscopic properties: Springer Verlag.

Wenzlau, F., and T. M. Müller, 2009, Finite-difference modeling of wave propagation and diffusion in poroelastic media: Geophysics, 74, no. 4 T55-T66, doi: 10.1190/1.3122928.

White, J. E., 1975, Computed seismic speeds and attenuation in rocks with partial gas saturation: Geophysics, 40, 224-232, doi: 10.1190/1 .1440520 .

White, J. E., N. G. Mikhaylova, and F. M. Lyakhovitsky, 1975, Low frequency seismic waves in fluid saturated layered rocks: Izvestija Academy of Sciences USSR, Physics of the Solid Earth, 11, 654-659. 